\title{
Diseño de un sistema móvil para la lectura de medidores mediante tecnología Bluetooth
}

\section{RESUMEN}

Actualmente en el Perú la lectura mensual de medidores de agua y de electricidad se realiza de forma manual, lo cual ocasiona pérdida de tiempo al hacer un doble registro: primero en la hoja manual y luego en el sistema de facturación; adicionalmente ocurren errores humanos de digitación, facturación mensual inexacta con la consiguiente insatisfacción de los clientes. Como una alternativa de solucion se plantea el diseño de un sistema móvil para la lectura remota de medidores, haciendo uso de tecnología Bluetooth.

Palabras clave: móvil, medidor, Bluetooth, lectura remota

DRAWING A MOBILE SYSTEM FOR METER READING THROUGH BLUETOOTH TECHNOLOGY

\section{ABSTRACT}

Currently in Peru, the water meter reading and electricity is conducted monthly by hand, resulting in loss of time to make a record twice: first in the manual page and then the billing system, additional human errors occur fingering, monthly billing with consequent inaccurate customer dissatisfaction. As an alternative solution arises designing a mobile system for remote meter reading, using Bluetooth technology.

Keywords: mobile, meter, Bluetooth, remote reading

\section{INTRODUCCIÓN}

La electricidad llegó a Lima en la antepenúltima década del siglo $\mathrm{XIX}$, luego de una historia de iluminación en base de lámparas de aceite, mecheros de kerosene y, a partir de 1857, se produce la iluminación a gas.

En 1906 se realizó la fusión de todas las empresas relacionadas con la industria eléctrica, formando las Empresas Eléctricas Asociadas, y en el año 1972, las Empresas Eléctricas Asociadas se constituyeron en ELECTROLIMA S.A.

Finalmente, en 1994, ELECTROLIMA se divide en tres nuevas empresas con el objetivo de su posterior privatización. De esta forma aparecen Luz del Sur, Edelnor y Edegel S.A., las dos primeras distribuidoras y la tercera generadora de electricidad para nuestra ciudad capital [2].

En la actualidad la lectura de medidores la realiza manualmente una persona casa por casa capturando la data. Posteriormente los datos son ingresados al computador. En esta última fase, cada operador se encarga de ingresar los datos que figuran en los reportes, correspondientes a 1000 ubicaciones técnicas, con más de 40000 medidores en total. Este proceso, toma entre tres y cinco días, y no está exento de cometer errores. Si la información no es correcta, se requiere enviar nuevamente una persona a revisar el medidor, causando demora en el proceso. Todo esto genera insatisfacción de los clientes y un costo adicional a la empresa.

El presente artículo presenta una alternativa de solución al problema planteando el diseño de un sistema móvil para la lectura de medidores, que permita obtener lecturas más exactas, haciendo uso de la tecnología Bluetooth.

\section{TECNOLOGÍA INALÁMBRICA}

El término "inalámbrico" hace referencia a la tecnología sin cables que permite conectar varias máquinas entre sí.

Inalámbrico significa que los dispositivos informáticos incorporan determinadas tecnologías (WLAN, GPRS, 3G, Bluetooth)

* Ingeniera en Sistemas e Informática UNMSM. E-mail: eli_saravia_16@hotmail.com

** Licenciada en Computación. Docente de la Facultad de Ingeniería de Sistemas e Informática UNMSM. E-mail: merruri@hotmail.com

*** Magíster en Computación e Informática UNMSM. Docente de la Facultad de Ingeniería de Sistemas e Informática UNMSM. E-mail: robertocalmet@gmail.com 
que permiten conectarse a otros dispositivos inalámbricos. Se pueden emplear estas conexiones inalámbricas para acceder al correo electrónico, utilizar una impresora inalámbrica para imprimir documentos desde una oficina remota o compartir información entre dispositivos, sin tener que preocuparse de cables ni de herramientas de interfaz física, permitiendo que la empresa aumente en eficacia y productividad.

\section{Los dispositivos inalámbricos utilizan tres} métodos de conexión principales:

\subsection{Bluetooth}

Bluetooth es una frecuencia de radio de disponibilidad universal que conecta entre sí los dispositivos habilitados para Bluetooth situados a una distancia de hasta 10 metros. Permite conectar un ordenador portátil o un dispositivo de bolsillo con otros ordenadores portátiles, teléfonos móviles, cámaras, impresoras, teclados, altavoces e incluso un ratón de ordenador.

\subsection{Wi-Fi}

Wi-Fi o red de área local inalámbrica (WLAN) es una red de $\mathrm{TI}$ de tamaño medio que utiliza la frecuencia de radio $802.11 \mathrm{a}, 802.11 \mathrm{~b}$ o $802.11 \mathrm{~g}$ en lugar de cables y permite realizar diversas conexiones inalámbricas a Internet. Si sabe dónde se encuentra una red Wi-Fi o WLAN, puede navegar por Internet, utilizar el correo electrónico y acceder a la red privada de su empresa.

\subsection{GPRS}

GPRS es la sigla de General Packet Radio Services (servicios generales de paquetes por radio). A menudo se describe como "2,5 G", es decir, una tecnología entre la segunda $(2 \mathrm{G})$ y la tercera $(3 G)$ generación de tecnología móvil digital. Se transmite a través de redes de telefonía móvil y envía datos a una velocidad de hasta $114 \mathrm{Kbps}$. El usuario puede utilizar el teléfono móvil y el ordenador de bolsillo para navegar por Internet, enviar y recibir correo, y descargar datos y soportes. Permite realizar videoconferencias con sus colegas y utilizar mensajes instantáneos para charlar con sus familiares y amigos, esté donde esté. Además, puede emplearse como conexión para el ordenador portátil u otros dispositivos móviles.

\subsection{G}

Al igual que GPRS, la tecnología 3G (tecnología inalámbrica de tercera generación) es un servicio de comunicaciones inalámbricas que le permite estar conectado permanentemente a Internet a través del teléfono móvil, el ordenador de bolsillo, el Tablet PC o el ordenador portátil. La tecnología 3G promete una mejor calidad y fiabilidad, una mayor velocidad de transmisión de datos y un ancho de banda superior (que incluye la posibilidad de ejecutar aplicaciones multimedia). Con velocidades de datos de hasta $384 \mathrm{Kbps}$, es casi siete veces más rápida que una conexión telefónica estándar.

Se dice que los usuarios de GPRS y $3 G$ están "siempre conectados", dado que con estos métodos de conexión tienen acceso permanente a Internet.

\section{TECNOLOGÍA MÓVIL}

La tecnología móvil permite llevar el trabajo a donde quiera que uno vaya (en el auto), en un avión, en el aeropuerto, en un restaurante o en el parque) y ofrece en todo momento la posibilidad de utilizar las aplicaciones instaladas, exponer presentaciones, crear documentos y datos, y acceder a ellos. Significa llevar siempre consigo el dispositivo que contiene toda su información y que le permite generar los documentos que necesita en todo momento y donde quiera que se encuentre.

\section{Diferencia tecnología inalámbrica y tecnología móvil}

Aunque ambos términos guardan relación con un aumento significativo de la productividad, existe una diferencia entre ellos. La diferencia es principalmente de enfoque: el término "móvil" hace referencia a la posibilidad de trasladar el trabajo de un sitio a otro, es decir, de llevar a cabo unas tareas determinadas sin estar físicamente en la oficina; en cambio, el término "inalámbrico" hace referencia a la posibilidad de conectar varios dispositivos entre sí o a una red sin necesidad de cables. (Según [HP])

\section{PROBLEMA}

Como puede observarse en la Figura 1, la tipología de reclamos sobre consumo es más abundante respecto de los reclamos por tarifa y ello ha contribuido a que el $92 \%$ de los casos esté referido a dicho elemento de la facturación, mientras que para el caso del segundo elemento, la tarifa, solo registra una tasa porcentual del $8 \%$ [TRASS, 2006].

\section{JUSTIFICACIÓN}

En la lectura de medidores de agua y electricidad se ve muchas deficiencias: entre ellas se tiene la demora en el proceso de lectura del medidor y los posibles errores de digitación y facturación. 
Es por ello que para facilitar los procesos que se realizan actualmente, se ha realizado la presente investigación que mediante el uso de una aplicación móvil con tecnología Bluetooth; reducirá el tiempo requerido para la lectura, así como también disminución del personal necesario para realizarlo.

- El costo de inversión del proyecto será aproximadamente:

Medidores electrónicos:

S/. $500 \mathrm{c} / \mathrm{u} *$ Clientes $=500 * 31205.3=15602650$

- Costos reducidos con el proyecto:

Reducción del número de trabajadores para la atención de reclamos:

$100 *$ Sueldo $=100 * 1000=100000$

Reducción de contrato de services para la toma de lectura S/. 500000

La inversión realizada en el proyecto se recuperaría en dos años aproximadamente, trayendo muchos beneficios para la empresa y a partir del tercer año empezará la ganancia proyectada, justificándose la inversión económica que requiere el proyecto, según el TIR.
Figura 1. Reclamos por consumo vs. Reclamos por tarifa por mes.

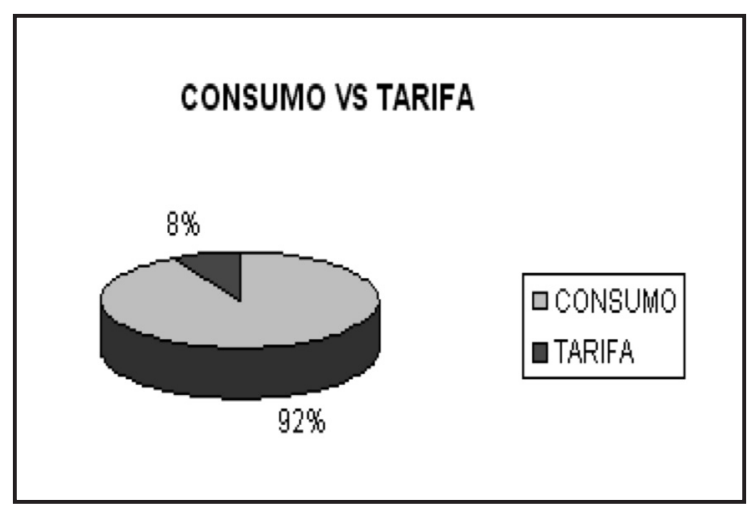

Fuente: TRASS, 2006

\section{ALCANCES}

Este trabajo de investigación podrá ser utilizado en el proceso de lectura de medidores de energía a través de un dispositivo móvil.

Figura 2. Mecanismos para la toma de lectura de medidores.

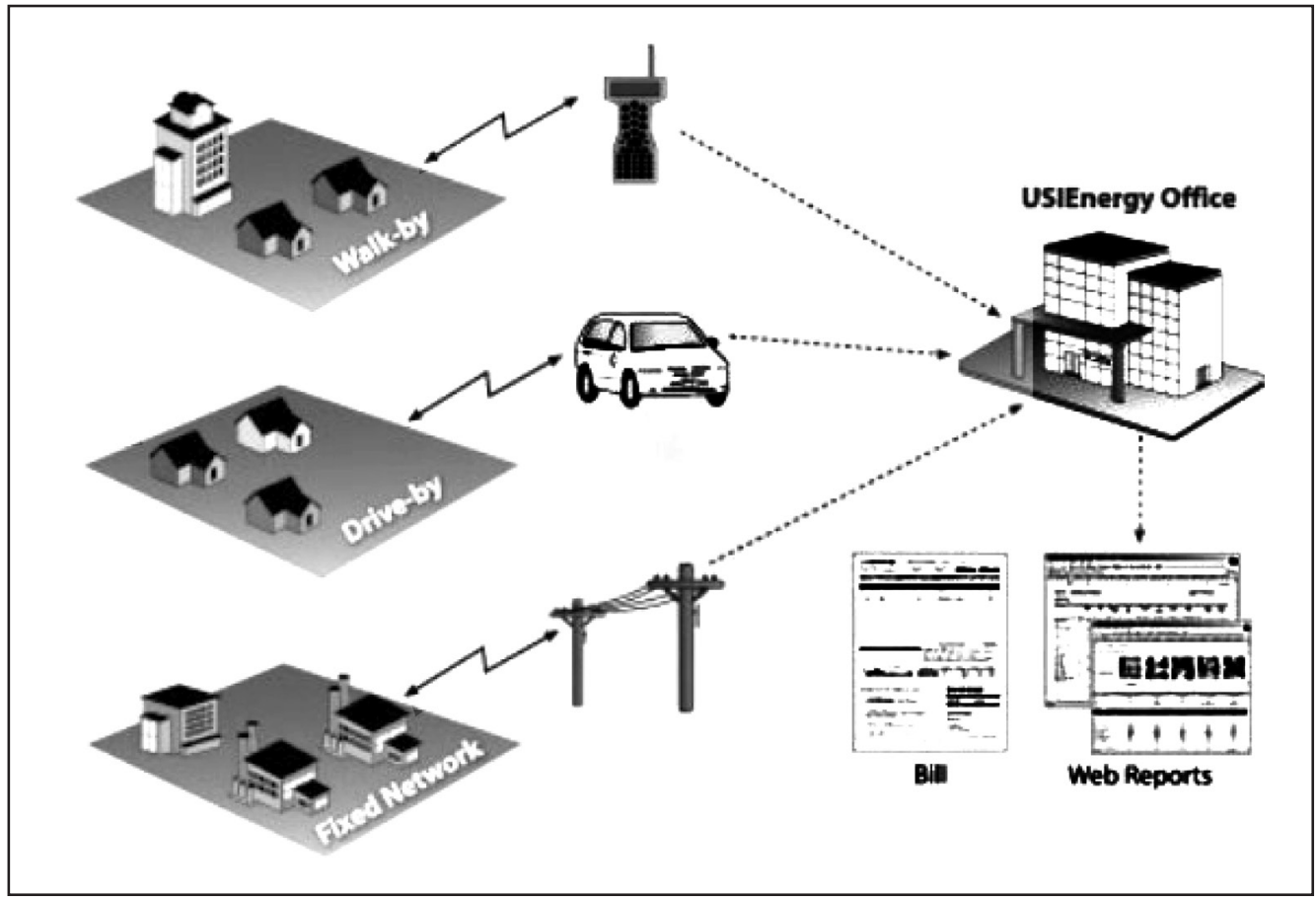

Fuente: Siemens et al., 2007. 


\section{CASOS DE ESTUDIO}

Como se muestra en la Figura 2, existen diversas formas de realizar la lectura remota de medidores, ya sea a pie con un dispositivo móvil, en automóvil o mediante un envío automático.

\section{Módulo Lectura Remoto (WAP)}

Este módulo utiliza la tecnología WAP que permite la toma de lectura de los medidores mediante un dispositivo móvil disminuyendo errores de digitación y tiempo del proceso de lectura. Así mismo para optimizar el tiempo de envío de información de la lectura de un medidor al servidor se utilizo la tecnología WML Script el cual realiza las validaciones en el mismo dispositivo móvil.

Actualmente en el Perú, los distritos que cuentan con esta nueva tecnología son: Distrito de Surquillo, distrito de Villa El Salvador, distrito de Comas y la provincia del Callao.

El dispositivo móvil utilizado es un celular Nextel en donde se ingresa manualmente las lecturas de los medidores de agua [Acros, 2005].

En la Figura 3, se muestra cómo se realiza el envío de información del operario hacia las bases de datos de los distrito de Surquillo, distrito de Villa El Salvador, distrito de Comas y la provincia del Callao, la cual se realiza mediante la tecnología Wap.

\section{Tecnología Bluetooth}

En la Figura 4, se muestra la metodología de facturación original, la cual propone la realización de la lectura del medidor y facturación al mismo tiempo, y evitar costos de personal para la entrega de recibos a los clientes.

A continuación se describe cada uno de los componentes de dicha propuesta:

Incluir la tecnología Bluetooth en el medidor le permite al operario tomar la lectura del mismo en lugares donde su acceso visual sería difícil. En este caso y aunque muy difundida, no es conveniente utilizar infrarrojo como interfaz de comunicación, dado su requerimiento de línea de vista entre dispositivos y corto alcance.

Adicionalmente, Bluetooth permite formar redes de hasta siete medidores los cuales pueden ser interrogados simultáneamente disminuyendo aun más el tiempo de lectura por operario.

La selección del teléfono celular como reemplazo del PDT, se debe a las funciones adicionales que actualmente traen estos dispositivos y la capacidad de la red celular para transferir datos. Algunas de las ventajas que resultan de utilizarlos son:

- Herramientas de programación completamente gratuitas que ayudan a su bajo costo.

- Su uso evita la necesidad de dotar a cada medidor con un MODEM GSM disminuyendo el costo del mismo.

- No se requiere un teléfono muy especializado y costoso, dado que el manejo y procesamiento grueso de la información se hace en el servidor.

Esta unión entre Bluetooth y la telefonía celular permite garantizar el acceso en línea de los datos del abonado eléctrico al momento de la lectura.

Figura 3. Lectura remota de medidores de agua.

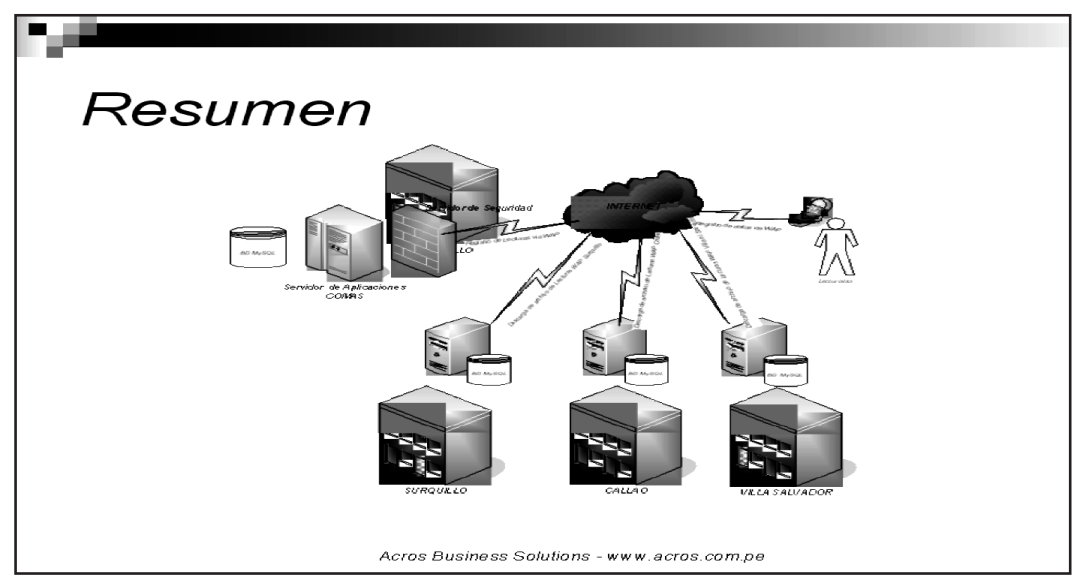


Figura 4. Lectura de medidores con tecnología Bluetooth.

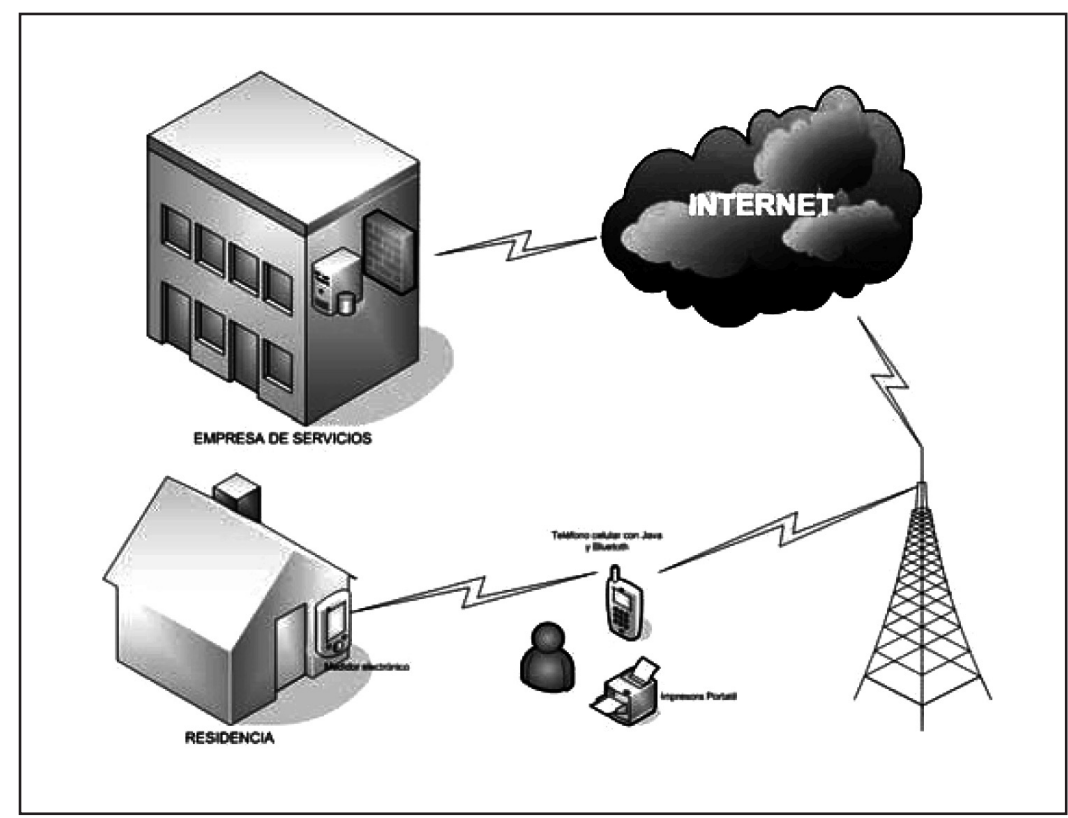

Fuente: Salazar et al, 2007.

\section{IMPLEMENTACIÓN}

La metodología a utilizar para el desarrollo, es el Proceso Unificado Rational (RUP), como se observa el modelo de negocio a través de la Figura 5 , Figura 6; el diagrama de actividades en la Figura 8; el diagrama de caso de uso obtener lectura en la Figura 12, y el diagrama de secuencia obtener lectura en la Figura 13. El lenguaje de programación es el Java, es decir, se programará el MIDlet que permita usar la tecnología Bluetooth con que cuentan los dispositivos móviles, de una manera amigable y fácil de manipular por parte del usuario, mostrado en las Figura 8, 9, 10 y 11.

\subsection{Modelo del negocio}

En el modelo del negocio se muestra cómo se realizan los procesos de negocio actualmente.

\section{a) Trabajadores del negocio:}

- Lecturista: Es la persona encargada de tomar la lectura a los medidores de las viviendas asignadas. Ver Figura 5.

- Digitadora: Es la persona encargada de realizar el ingreso de información de las lecturas obtenidas por los lecturistas al sistema informático de la empresa. Ver Figura 6.
Figura 5. Actor lecturista.

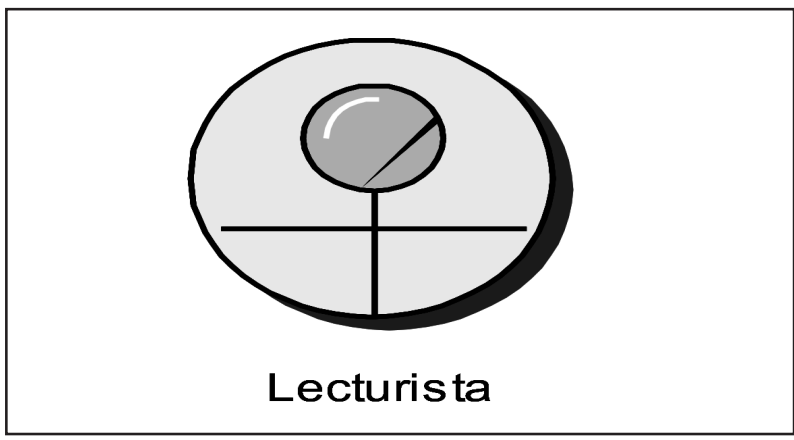

Fuente: Elaboración propia.

Figura 6. Actor digitador.

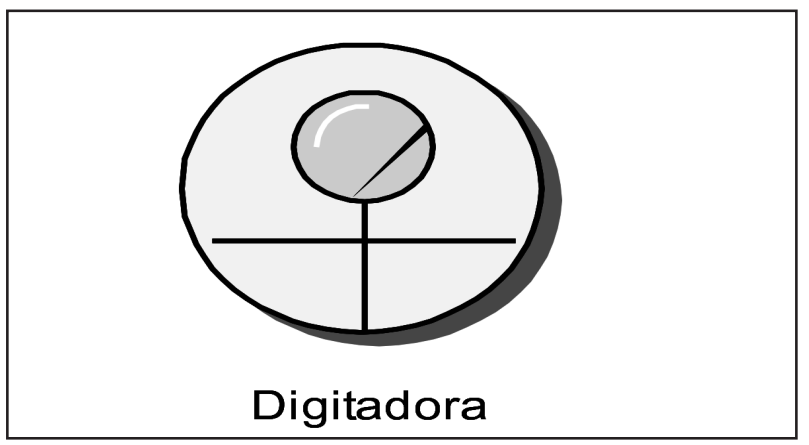

Fuente: Elaboración propia. 


\section{b) Diagrama de actividades}

A continuación mostraremos:

Figura 7. Diagrama de actividades del proceso de lectura.

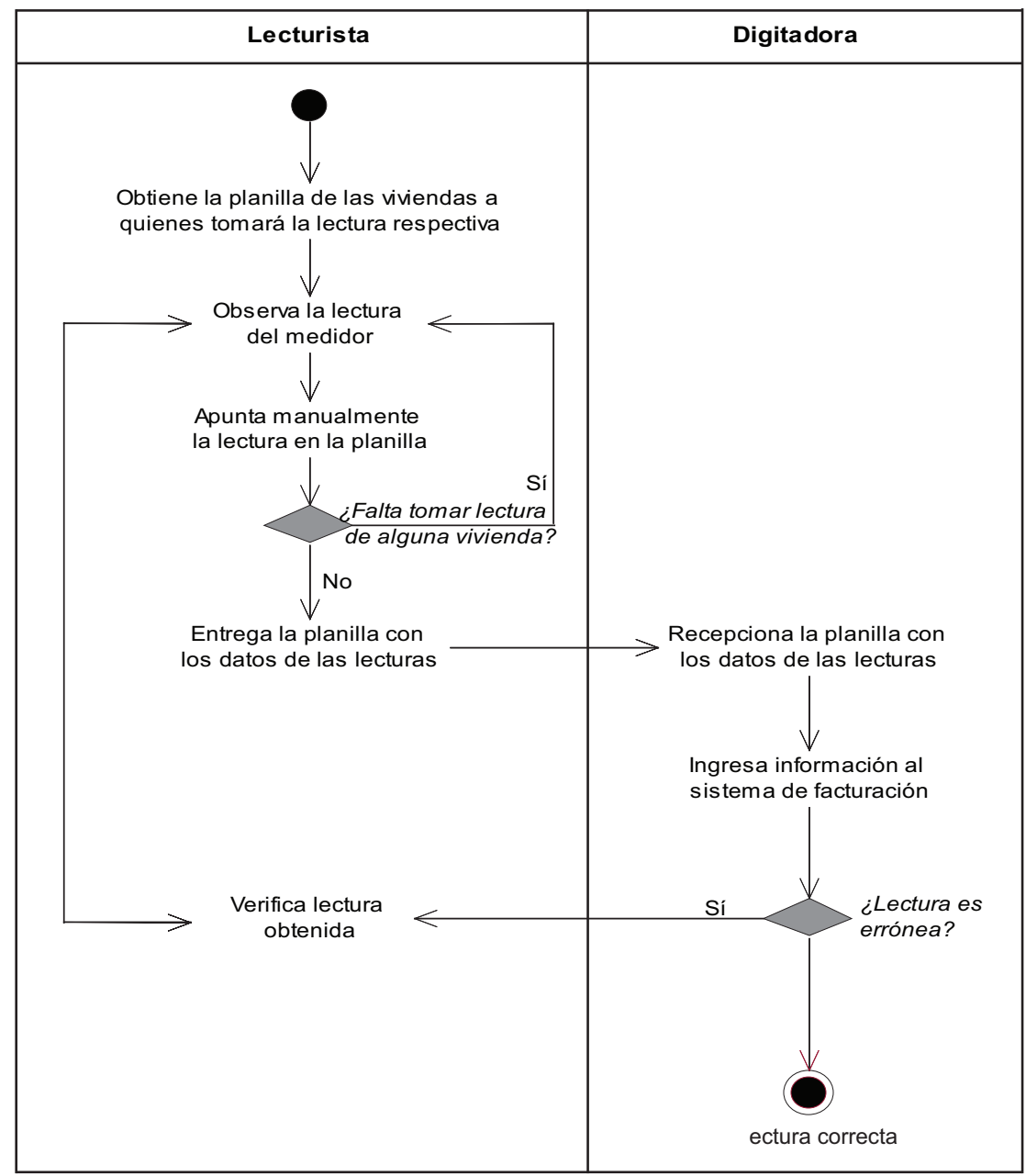

Fuente: Elaboración propia.

\section{PROTOTIPOS}

Figura 8. Prototipos de ingreso al sistema.
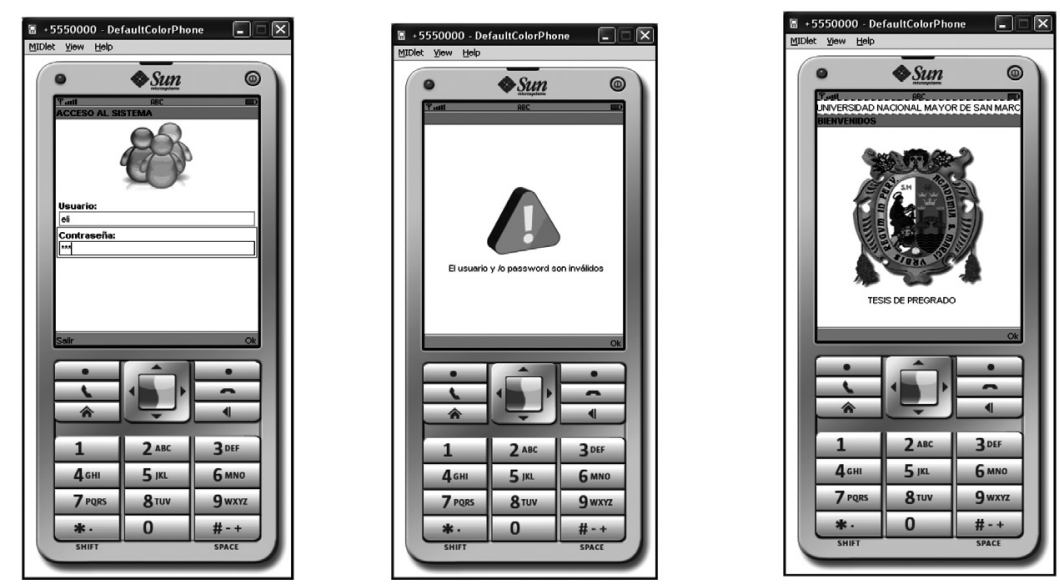


\section{Lectura automática}

Figura 9. Prototipos de lectura.

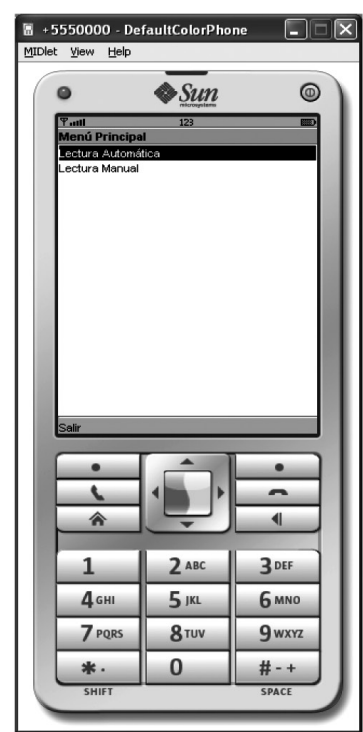

MENÚ PRINCIPAL

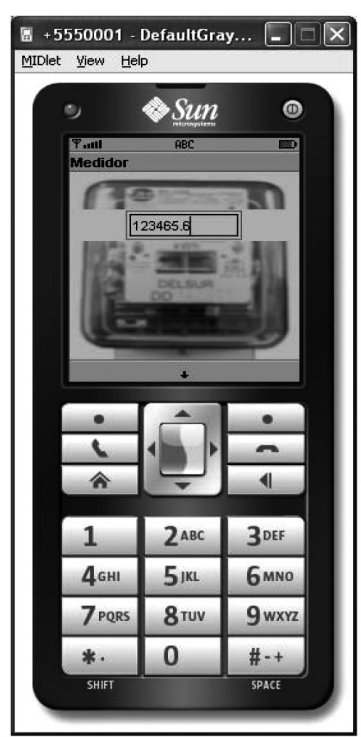

SIMULADOR MEDIDOR

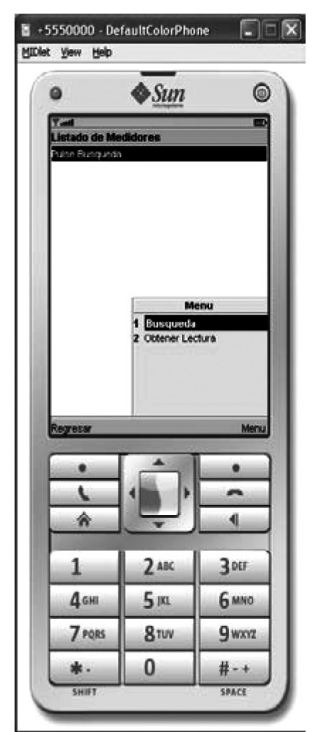

MENÚ DE OPCIONES

Fuente: Elaboración propia.

\section{Búsqueda}

Figura 10. Prototipo de búsqueda.

LISTADO DE MEDIDORES

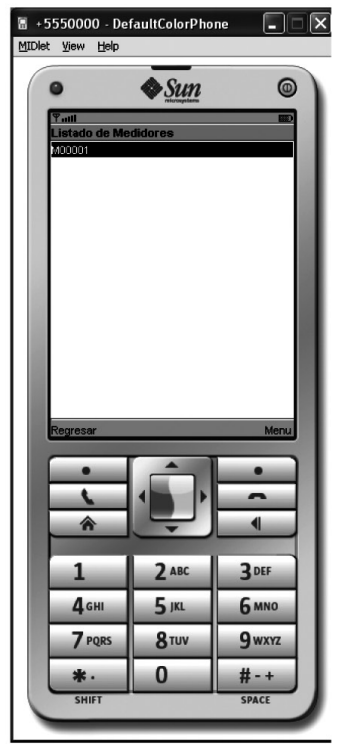

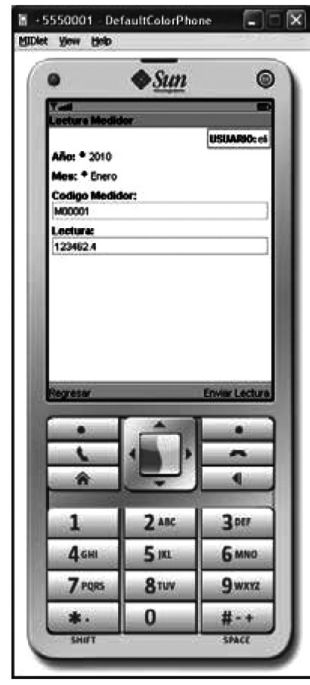

Obtener Lectura

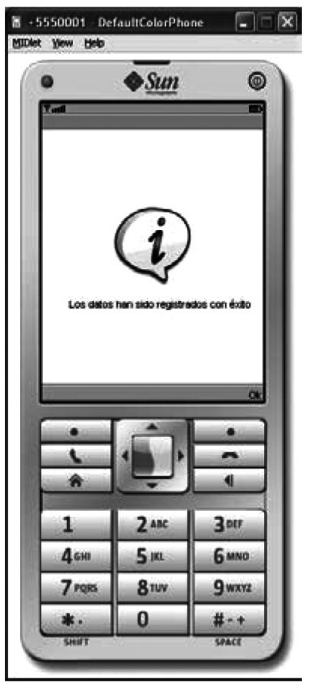

Enviar Lectura

Fuente: Elaboración propia. 


\section{Lectura manual}

Figura 11. Lectura manual.

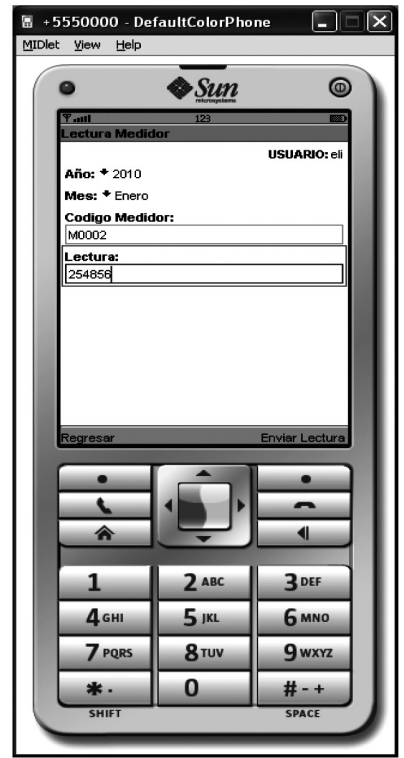

Obtener Lectura

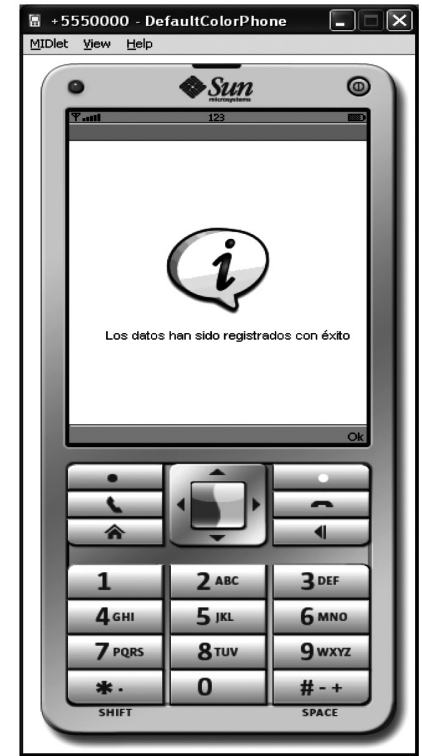

Enviar Lectura

Fuente: Elaboración propia.

Modelo de Análisis:

Caso de uso: Obtener lectura

MODELO DE ANÁLISIS:

Figura 12. Caso de uso obtener lectura.

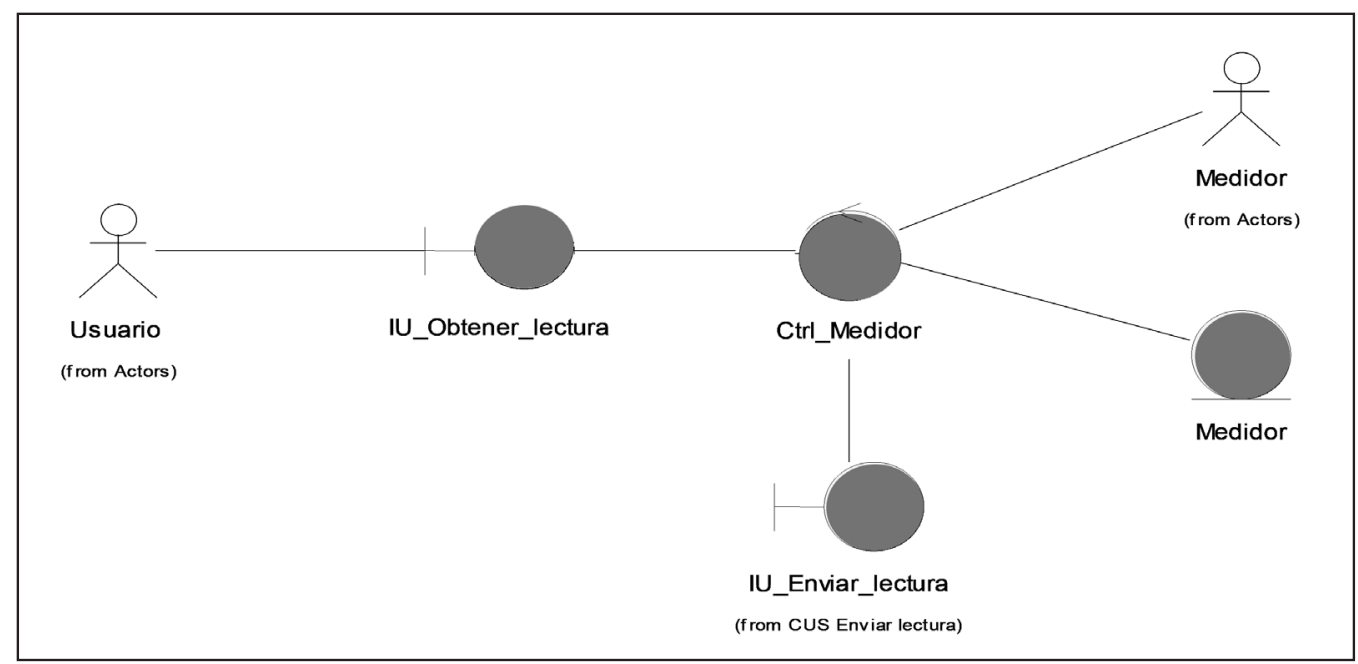

Fuente: Elaboración propia. 


\section{DIAGRAMA DE SECUENCIA:}

Figura 13. Diagrama de secuencia obtener lectura.

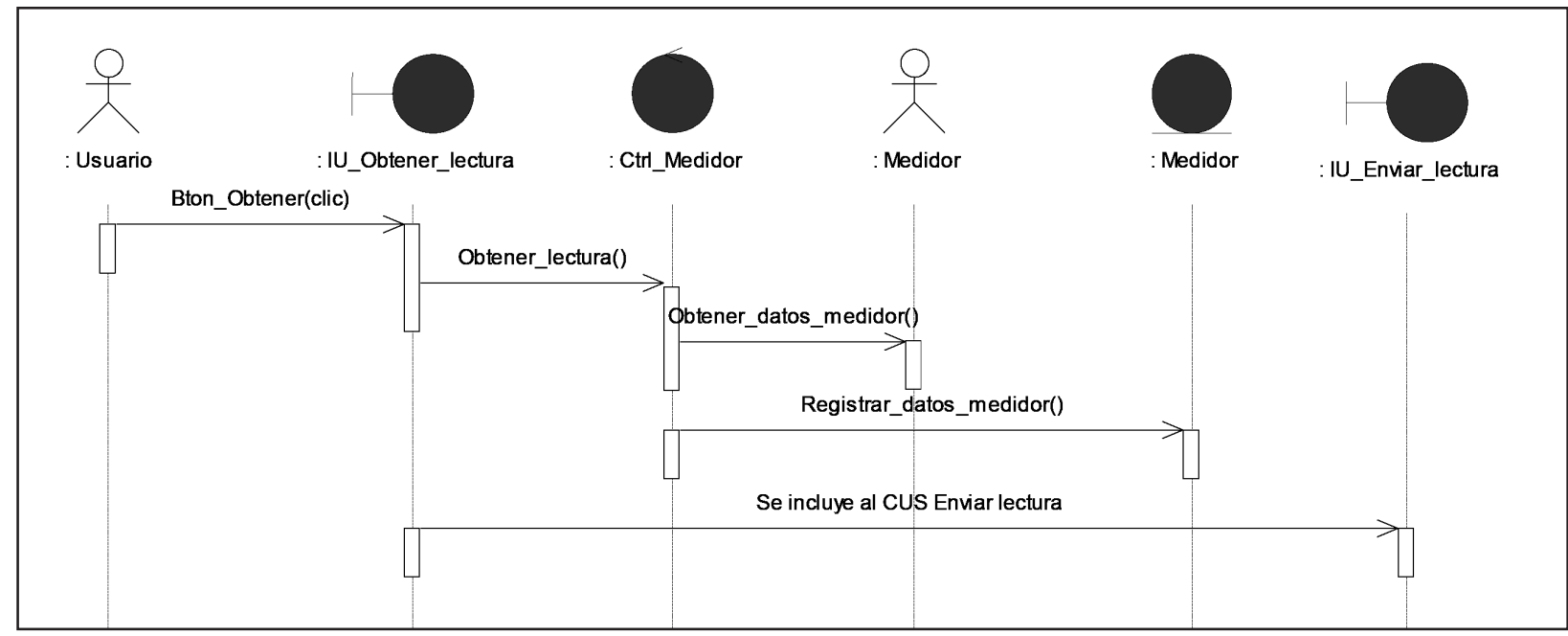

Fuente: Elaboración propia.

\section{ARQUITECTURA DEL SISTEMA}

La metodología presentada propone mejorar el proceso de lectura de los medidores, teniendo en cuenta el cambio de los medidores electromecánicos que se usan actualmente por los medidores electrónicos.

En la Figura 14 se muestra la arquitectura propuesta para el desarrollo del sistema móvil, el cual obtendrá la lectura automática del medidor electrónico y enviará los datos capturados hacia el sistema central de facturación de la empresa prestadora de servicios, evitando posibles errores de digitación y disminuyendo el tiempo de lectura de medidores.

\section{- Medidor electrónico inalámbrico:}

El nuevo medidor de energía debe utilizar alguna interfaz de comunicación inalámbrica de bajo costo, que permita mediante algún otro dispositivo de tipo portátil, obtener automáticamente el consumo de energía y el número de matrícula de cada medidor, eliminando la posibilidad de errores en la lectura o error humano.

\section{- Reemplazo de registro manual:}

Se requiere un nuevo dispositivo de bajo costo que a diferencia del registro manual permita la conexión inalámbrica con el medidor y la base de datos de la empresa de servicios, de tal forma que se tenga información en línea de los clientes.

\section{CONCLUSIONES Y RECOMENDACIONES}

- Este proyecto ha permitido dar un uso adicional a la tecnología Bluetooth integrada en los teléfonos celulares. También se ha demostrado que la plataforma java ME creada para dispositivos móviles se puede usar para controlar un sistema Bluetooth en teléfonos celulares.

- Se ha conseguido desarrollar un sistema móvil con tecnología inalámbrica Bluetooth sencillo y a la vez muy útil.

- Dentro del mercado actual existen muchas tecnologías para control remoto tanto a nivel doméstico como industrial. Se puede concluir para control remoto tanto a nivel doméstico como industrial. Se puede concluir que Bluetooth es un ejemplo del avance acelerado de la tecnología para integrar las telecomunicaciones globalmente y proveer acceso a más personas día a día.

- La tecnología Bluetooth es muy difundida en el área de los teléfonos celulares, cada vez más modelos más la incluyen por lo que el campo de aplicaciones del proyecto sigue creciendo. Por esta razón, en domótica como ZigBee e inclusive WiFi, se puede concluir que el usar un teléfono celular como dispositivos de control es muy ventajoso, puesto que ya tiene incluida esta tecnología. 
Figura 14. Arquitectura del Sistema.

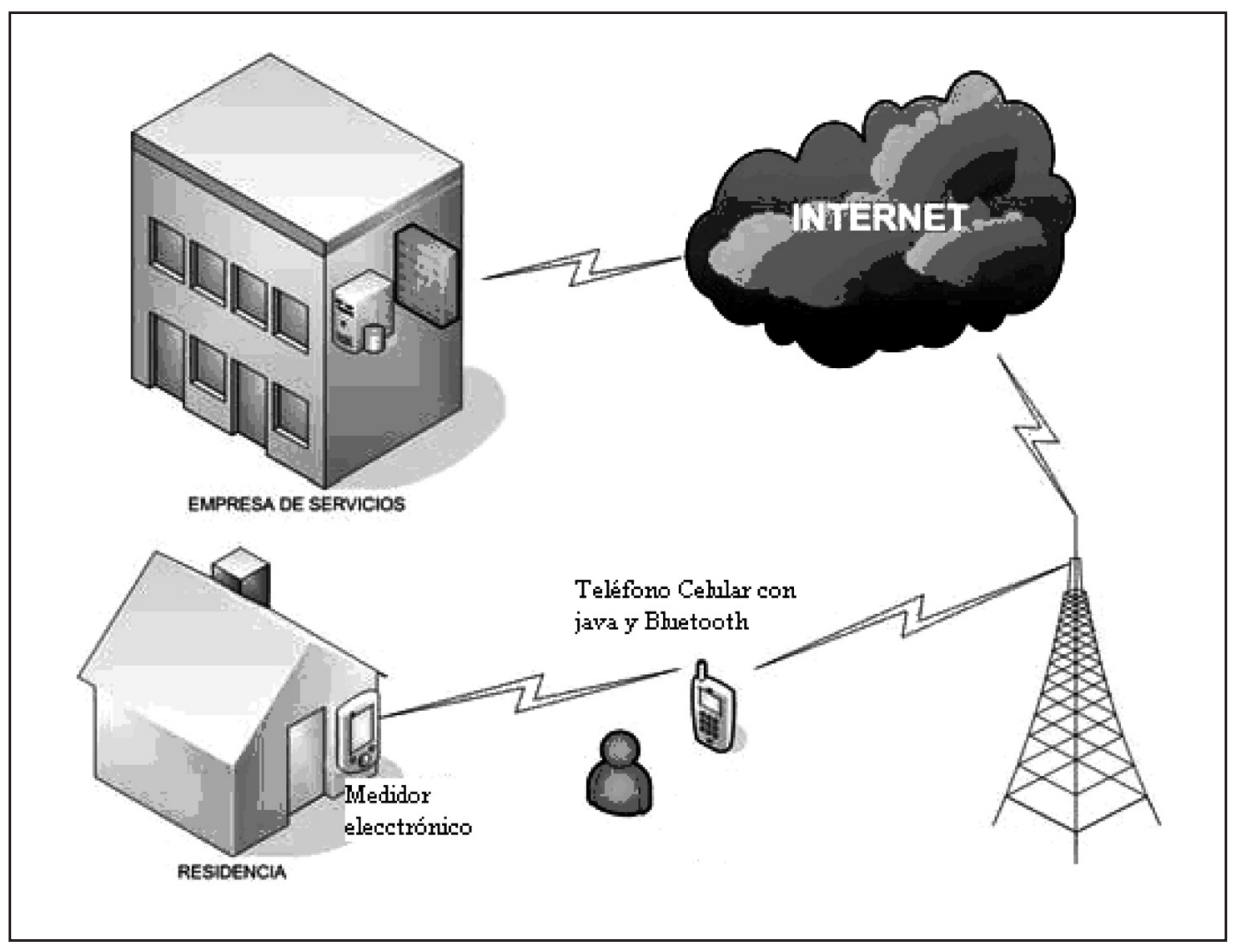

Fuente: Elaboración basado en Salazar et al., 2009.

\section{REFERENCIAS BIBLIOGRÁFICAS}

\section{Tesis:}

[1] Palacios et al. (2006). Sistema de Localización y Consulta de Servicios por celular haciendo uso de la tecnología inalámbrica, Tesis, Universidad Nacional Mayor de San Marcos, Perú.

\section{Revistas:}

[1] Chaves et al. (2008). Chaves Osorio José Andrés, Cano Garzón Hugo Baldomero, Elias Giraldo Esteban, "Medición del Consumo de Agua Domiciliaria Utilizando Tecnologia Inalámbrica Zigbee", Scientia Et Technica, Vol XIV, pp.43-47.

[2] Marc et al. (2008). "A tabu search algorithm for the global planning problem of third generation mobile networks", Computers and Electrical Engineering, Vol. 34, pp. 470-487, 2008

[3] Salazar et al. (2007). "Nueva Arquitectura Abierta De Tiempo Real Para La Facturación Insitu De Abonados Eléctricos", Scientia et Technica, vol XIII, pp. 117-122.

\section{Direcciones electrónicas:}

[1] Acros Business Solutions, "Sistema Integrado de Servicios", http://www.acros.com.pe
[2] ELECTROPERU S. A. (2000). ELECTROPERU S. A., "Electricidad en el Perú", Museo de la Electricidad, http://museoelectri.perucultural. org.pe/central4.htm, Junio 2000

[3] HP, ¿Qué es la tecnología inalámbrica?",http:// h41320.www4.hp.com/cda/mwec/display/ main/mwec_content.jsp? zn=hpsmb \& $\mathrm{cp}=26-29-31-30-37^{\wedge} 3042 \_4003 \_10$

[4] Ministerio de Energía y Minas (2003). "Distribución de Energía Eléctrica", Ministerio de Energía y Minas.

[5] Progress Energy (2005). Progress Energy, "Medidores Automáticos", http://www.progressenergy.com/community/espanol/medidores.asp

[6] Siemens et al. (2007). Siemens, Neptuno Technology Group, "Telelectura de medidores de energía con tecnologías AMR y AMI", Afinidad Eléctrica, http://www.afinidadelectrica. com/articulo.php?IdArticulo=124, 2007

[7] TRASS (2006). TRASS (Tribunal Administrativo de Solución de Reclamos de los Usuarios de Servicios de Saneamiento), "Boletín del Trass", http://www.sunass.gob.pe/doc/trass/boletin2/ diseno_boletin.htm, Julio 2006 